\title{
Large-scale genomic correlations in Arabidopsis thaliana relate to chromosomal structure
}

\author{
Wayne S Kendal*1,2 and Brian P Suomela ${ }^{3}$
}

\author{
Address: ${ }^{1}$ Division of Radiation Oncology, The Ottawa Hospital Regional Cancer Centre, Ottawa, Ontario, K1H 1C4 Canada, ${ }^{2 T h e}$ Ottawa \\ Hospital Research Institute, Ottawa, Ontario, K1H 8L6 Canada and ${ }^{3}$ Ontario Genomics Innovation Centre, Ottawa, Ontario, K1H 8L6 Canada \\ Email: Wayne S Kendal* - wkendal@ottawahospital.on.ca; Brian P Suomela - briansuomela@gmail.com \\ * Corresponding author
}

Published: 02 June 2005

BMC Genomics 2005, 6:82 doi:10.1 |86/|47|-2/64-6-82

This article is available from: http://www.biomedcentral.com/I47I-2164/6/82

(c) 2005 Kendal and Suomela; licensee BioMed Central Ltd.

This is an Open Access article distributed under the terms of the Creative Commons Attribution License (http://creativecommons.org/licenses/by/2.0), which permits unrestricted use, distribution, and reproduction in any medium, provided the original work is properly cited.
Received: 27 March 2005

Accepted: 02 June 2005

\begin{abstract}
Background: The chromosomes of the plant Arabidopsis thaliana contain various genomic elements, distributed with appreciable spatial heterogeneity. Clustering of and/or correlations between these elements presumably should reflect underlying functional or structural factors. We studied the positional density fluctuations and correlations between genes, indels, single nucleotide polymorphisms (SNPs), retrotransposons, 180 bp tandem repeats, and conserved centromeric sequences (CCSs) in Arabidopsis in order to elucidate any patterns and possible responsible factors for their genomic distributions.

Results: The spatial distributions of all these elements obeyed a common pattern: the density profiles of each element within chromosomes exhibited low-frequency fluctuations indicative of regional clustering, and the individual density profiles tended to correlate with each other at large measurement scales. This pattern could be attributed to the influence of major chromosomal structures, such as centromeres. At smaller scales the correlations tended to weaken - evidence that localized cis-interactions between the different elements had a comparatively minor, if any, influence on their placement.

Conclusion: The conventional notion that retrotransposon insertion sites are strongly influenced by cis-interactions was not supported by these observations. Moreover, we would propose that large-scale chromosomal structure has a dominant influence on the intrachromosomal distributions of genomic elements, and provides for an additional shared hierarchy of genomic organization within Arabidopsis.
\end{abstract}

\section{Background}

With recent advances in molecular biology we have begun to appreciate both the diversity of genomic elements contained within chromosomes, and the complexity of their distribution. For example, within plant chromosomes there is often a centromeric region of tandemly repeated satellite sequences that is flanked by transposons, retroelements, middle-repetitive elements, and pseudogenes.
Beyond this, the genes tends to become more concentrated $[1,2]$. Other genomic elements like SNPs tend to form smaller scale-invariant clusters within chromosomes, a finding presumably attributable to the random and independent assortment of haplotype blocks, each with distinctive genealogical histories[3,4]. Genes exhibit a clustering similar to SNPs, although the mechanisms at play here are less well understood[5]. Housekeeping 
genes cluster differently, within regions of high GC content $[6,7]$, and there is evidence that other co-expressed genes cluster within specific chromosomal domains[8].

There are other examples of such non-random positional associations within chromosomes. For example, indels have been reported to collocate with SNPs[9], and transposable elements with regions of low gene density[10]. Eukaryotic genomes appear to be both structurally and functionally organized at multiple hierarchical levels, and these non-random patterns presumably are manifestations of this organization[11].

We performed a detailed analysis of the intrachromosomal distributions of 6 different genomic elements within the Arabidopsis genome. In addition to genes, indels, SNPs, and retrotransposons, we studied a number of plant-specific elements catalogued through The Arabidopsis Information Resource (TAIR)[12], The Institute for Genomic Research (TIGR)[13] and the Arabidopsis genomic repeat database, AtRepBase. This included a class of $180 \mathrm{bp}$ tandem repeats found in the Arabidopsis genome[1] and provided by AtRepBase, as well as a class of CCSs as identified by The Arabidopsis Genome Initiative [14-19] and catalogued within the TIGR plant repeat database.

\section{Results}

Figure 1 provides the density profiles of these elements within chromosome 1, as enumerated from both 50 and $1000 \mathrm{~kb}$ bins. The smaller bins revealed numerous highfrequency fluctuations; the larger bins more smoothed density fluctuations. In several of the smoothed profiles there were comparatively large density fluctuations coincident with their known positional associations with centromeres[1]. The profiles corresponding to indels and SNPs, however, did not reveal such major centromeric associations. Parenthetically, we should mention that there remain relatively large gaps in the Arabidopsis centromeric regions that have not been completely defined.

We performed spectral analyses of these profiles. Briefly, spectral analysis is a computational method used to study data that fluctuates about a mean value over either time or position. It is based on the mathematical theory of Fourier series, and allows one to resolve density fluctuations like those we observed here into their component harmonics. For each set of density fluctuations we were able to deduce a spectrum, where the intensity (or power) of each harmonic could be plotted $v$ s. its particular frequency. Such power spectra can often be exploited to characterize the underlying mechanisms of the fluctuations.

Figure 2 gives the power spectrum for each genomic element, averaged over all 5 chromosomes. Remarkably, each different spectrum shared a qualitatively similar pattern. The greatest intensity of fluctuation was at the lowfrequency end of each spectrum. This low-frequency activity corresponded to the large-scale fluctuations apparent to the smoothed density profiles (Fig. 1). (It would be helpful remind the reader here that frequency in these spectra was inversely related to the scale of the measurement bin size.) Further inspection of these density profiles revealed that the centromeric region had provided a major contribution to these low-frequency density fluctuations for genes, retrotransposons, 180 bp repeats and CCSs.

The low-frequency activity associated with indels and SNPs was admittedly less intense than that observed with the other elements. Yet the highest peaks from each of these power spectra were both qualitatively similar to those demonstrated with the other genomic elements, and distinguishable from simulated random background noise $(P<0.006)$. On the basis of the qualitative similarity between the 6 different power spectra we concluded that the major underlying process(es) that governed the density distributions of the different genomic elements had a similar stochastic basis.

These low-frequency density fluctuations, as observed from 6 genomic elements, indicated a non-random clustering of the individual elements over comparatively large chromosomal regions. Since the indels and SNPs had not exhibited such major concentration changes in the centromeric regions, and since genes, indels, SNPs, and retrotransposons had exhibited low-frequency density fluctuations in non-centromeric regions (Fig. 1), we concluded that non-centromeric chromosomal features may have also contributed to the low frequency activity.

Next we sought to determine whether local concentrations of the different elements might correlate with each other. We calculated the Pearson correlation coefficient $r$ between the paired density profiles of the different elements. Given 6 elements, this yielded 15 different permutations. Table 1 provides $r$ for all these different permutations, as assessed at $1 \mathrm{Mb}$ intervals. Thirteen of these correlations were statistically significant. Six exceeded $|r|>0.6$ and could be thus considered relatively strong.

Statistically significant and strong correlations between the positions of indels and SNPs were apparent from our analysis, in agreement with observations from other systems[9]. We also observed a statistically significant and strong negative correlation between genes and retrotransposons, a finding similar to that reported between genes and transposable elements[10]. 
a.

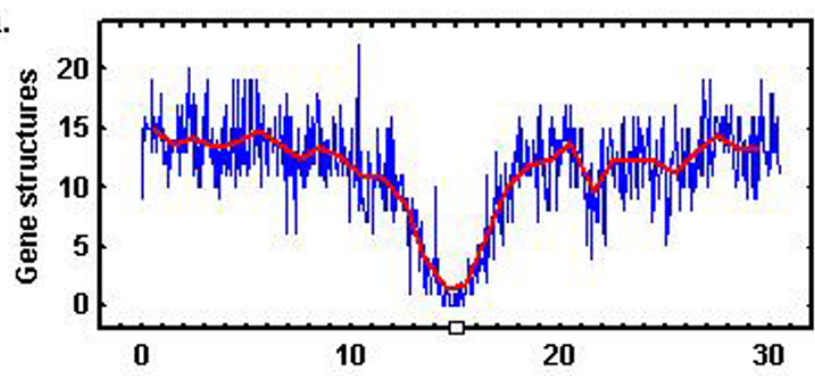

b.

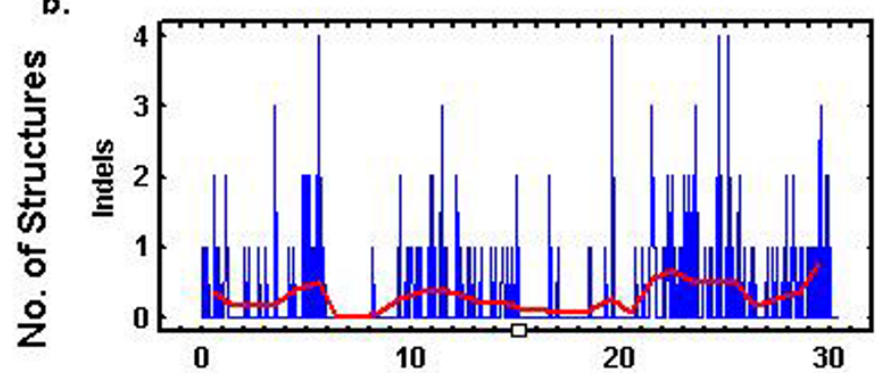

c.

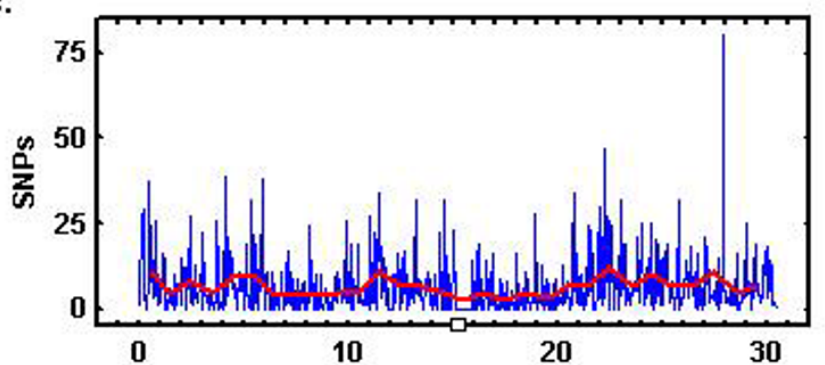

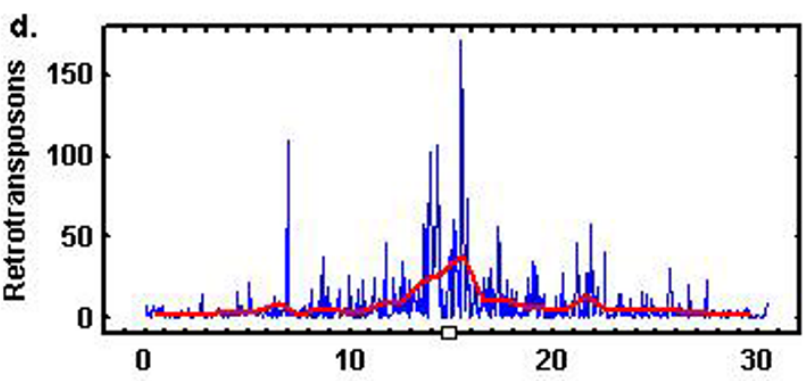

e.

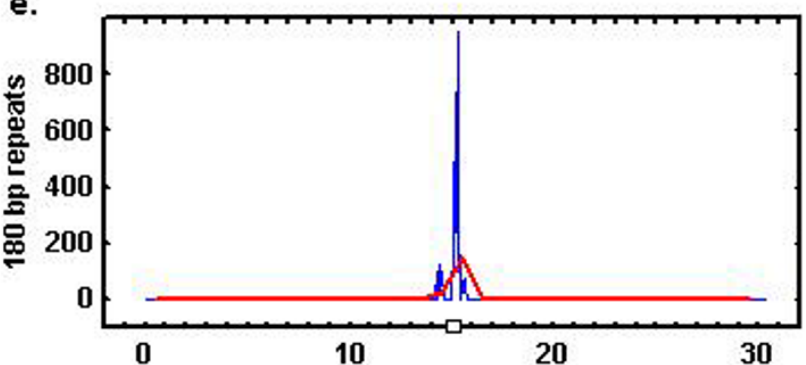

f.

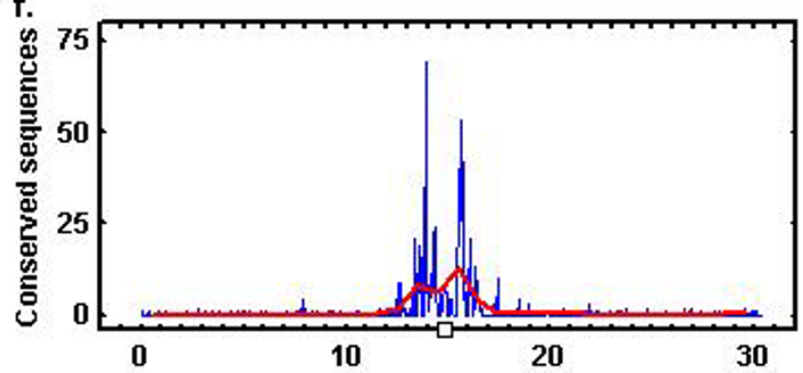

Physical Distance (Mb)

Figure I

Density profiles for genomic elements along the length of Arabidopsis chromosome I. Local densities were estimated based upon $50 \mathrm{~kb}$ (blue lines) and I Mb (red lines) enumerative bins and the density profiles were plotted as the number of structures per $50 \mathrm{~kb}$ length of chromosome vs. physical distance as measured from the p-terminal of the chromosome. (a) Genes. (b) Indels. (c) SNPs. (d) Retrotransposons. (e) 180 bp repeats. (f) CCSs. The centromeric region was located at about $15 \mathrm{Mb}$ from the $\mathrm{p}$-terminus. The discontinuity indicated along each $\mathrm{X}$-axis is indicative of the gap in the physical map at this region. Although the lines and axes are drawn continuously in these graphs we must remember that there remains large gaps within the centromeric regions of each chromosome which have not been completely defined.

We then sought to determine how changes in measurement scale might affect $r$. In Fig. 3, several of the strongest correlations are plotted over a range of measurement scales. Each of these relationships was statistically significant for the full range of scale. Five of these correlation profiles, however, showed marked weakening $(r \leq 0.2)$ at bin sizes below $200 \mathrm{~kb}$. The remaining correlation profile, between indels and SNPs (Fig. 3b), however did not reveal as pronounced a decrease at the lower bin sizes. This latter case was an exception to a general trend - the envelope from all 15 correlation pairs (Fig. 3, insert) revealed that the majority of correlations weakened considerably, yet remained statistically significant, at the smaller scales. Because these correlations tended to be strongest at large scales, the associations between elements implied by these correlations were presumably relatively longranged. Localized cis-interactions thus did not appear to have a major influence on the intrachromosomal distribution of these elements.

\section{Discussion}

To summarize, the density profiles of the 6 diverse genomic elements examined here revealed numerous high frequency and localized fluctuations upon which 


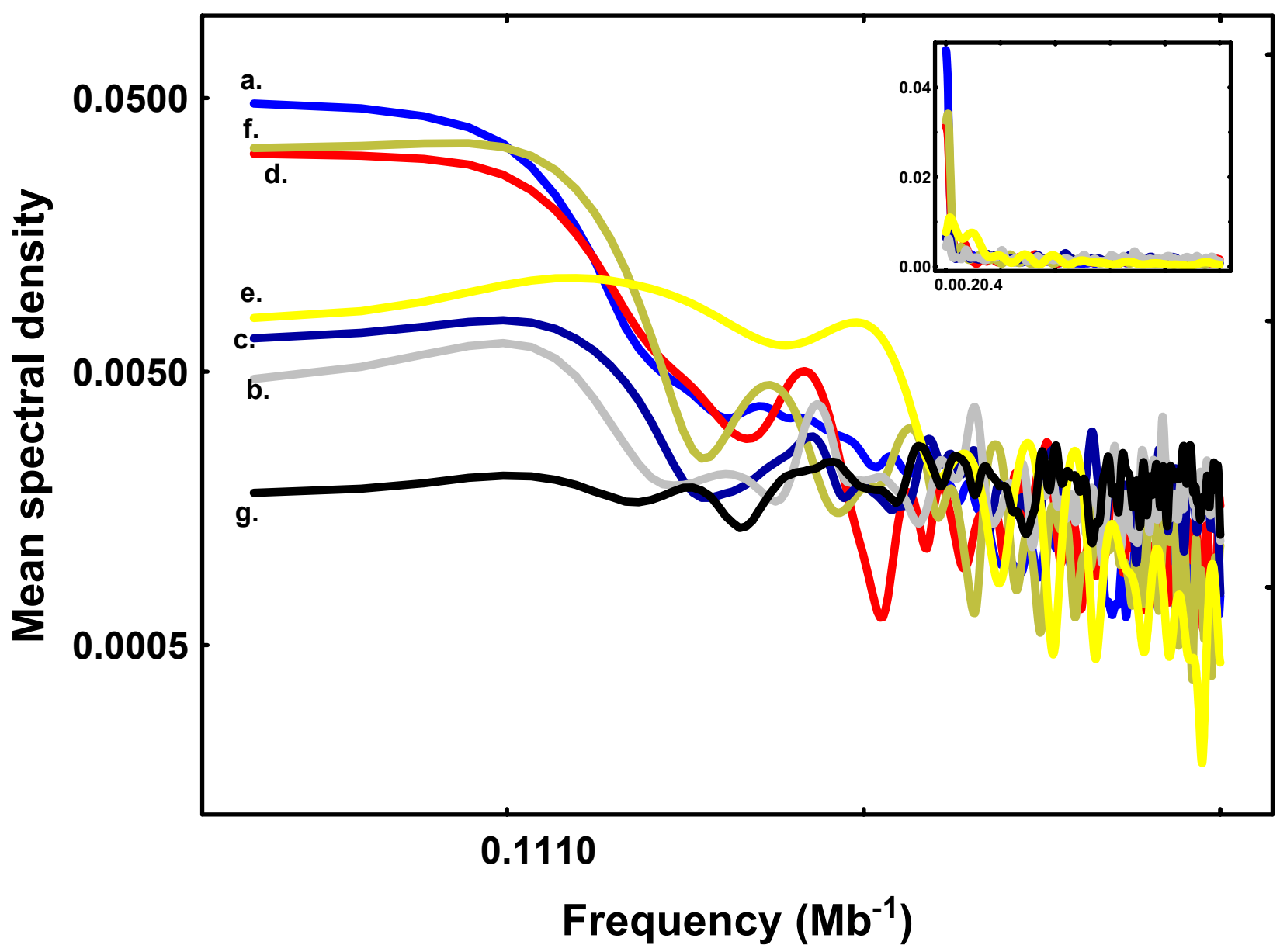

Figure 2

Power spectra for the fluctuations in positional density from genomic elements in Arabidopsis chromosomes. Mean spectral densities for the fluctuations of each element within individual chromosomes were calculated, normalized, averaged over all 5 Arabidopsis chromosomes, and then plotted vs. frequency. The power spectra for all 6 genomic elements were qualitatively similar on this log-log plot: the most intense fluctuations were located at the low-frequency ends of the spectra. (a) Genes. (b) Indels. (c) SNPs. (d) Retrotransposons. (e) 180 bp repeats. (f) CCSs. (g) Simulated data from a Poisson distribution. (Insert) Mean Spectral Densities plotted with linear scales to emphasize the concentration of density fluctuations at low frequency.

was superimposed a low frequency and large scale pattern of fluctuation. For 4 of these elements these low frequency fluctuations were concentrated at the centromeric regions of the chromosomes. Spectral analysis confirmed the presence of a dominant low frequency, large scale, component to these density fluctuations in each of the 6 elements, and these fluctuations tended to correlate with each other at large genomic scales. It was apparent on comparative analysis of these three sets of figures that the large scale density fluctuations seen within the density profiles of Fig. 1 were related to the dominant low frequency components evident from the power spectra of Fig. 2, and to the relatively strong large scale correlations seen within Fig. 3. It was also reasonably obvious from Fig 1 that a major (but not exclusive) component to these large scale density fluctuations was derived from centromeric structures. 
Table I: Correlations between different genomic elements in Arabidopsis.

\begin{tabular}{lll}
\hline Genomic elements compared & $r^{\mathrm{a}}$ & $P$ value $^{\mathrm{b}}$ \\
\hline retrotransposons vs. CCSs & 0.87 & $<0.0000 \mathrm{I}$ \\
retrotransposons vs. I80 bp repeats & 0.75 & $<0.0000 \mathrm{I}$ \\
indels vs. SNPs & 0.68 & $<0.0000 \mathrm{I}$ \\
I80 bp repeats vs. CCSs & 0.66 & $<0.0000 \mathrm{I}$ \\
genes vs. retrotransposons & -0.65 & $<0.0000 \mathrm{I}$ \\
genes vs. CCSs & -0.63 & $<0.00001$ \\
SNPs vs. retrotransposons & -0.46 & 0.00005 \\
SNPs vs. CCSs & -0.42 & 0.0004 \\
genes vs. I80 bp repeats & -0.41 & 0.001 \\
indels vs. CCSs & -0.40 & 0.001 \\
SNPs vs. I80 bp repeats & 0.40 & 0.002 \\
indels vs. retrotransposons & -0.32 & 0.03 \\
indels vs. I80 bp repeats & -0.31 & 0.05 \\
genes vs. SNPs & 0.30 & $\mathrm{NSc}$ \\
genes vs. indels & 0.23 & $\mathrm{NS}$ \\
\hline
\end{tabular}

athe correlation coefficient $r$ given here represents the quadratic mean taken from all five Arabidopsis chromosomes using I Mb enumerative bins; ${ }^{\mathrm{P} P}$ value, probability value; $\mathrm{C} N \mathrm{~S}$, not significant;

These large scale correlations were demonstrated with several different genomic elements, which were themselves identified and mapped through an assortment of different methods. The possibility that some form of selection bias might have influenced the results should be considered. It would, however, be difficult to postulate a spurious source of bias to explain such fluctuations and correlations for which the predominant component was evident not only at low frequency and large scales but also with each of the different elements examined. Such a putative bias would necessarily have to be associated with the centromeres. Despite the large undefined gaps in centromeric regions that remain, sufficient portions of the centromeres have been defined so as to provide us with strong evidence for the associated centromeric density changes for gene structures, retrotransposons, 180 bp repeats and conserved centromeric sequences[1,2]. We would propose instead that the most plausible explanation for these low frequency density fluctuations, and large scale correlations, would be itself the large scale structural features of Arabidopsis chromosomes.

The persistent correlation between indels and SNPs at smaller measurement scales (Fig. 3c) warrants further consideration. These two elements have been shown to correlate with each other within the HLA region of the human genome, a observation that might be attributed to repetitive insertions and deletions, imperfect segmental duplications or adjacent nucleotide changes[20] - all of these presumably localized processes. Our observation of a modest $20 \%$ increase in the respective value of $r$ with

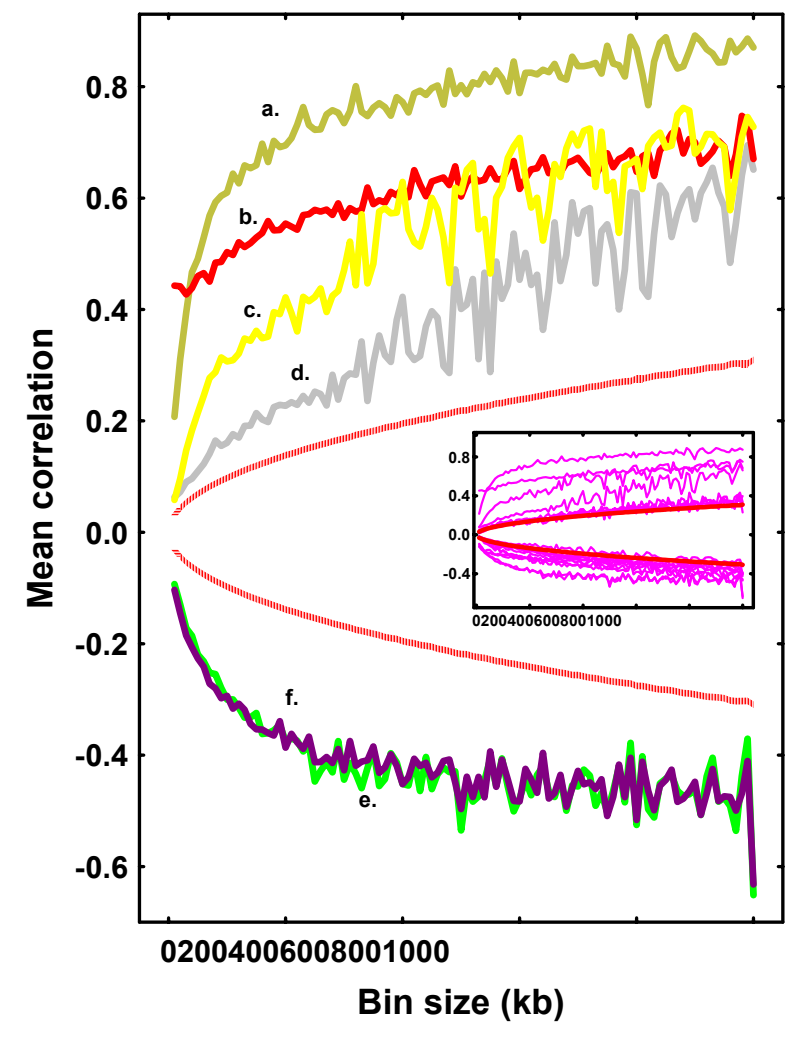

Figure 3

Mean correlation coefficient vs. measurement bin size. The quadratic mean of $r$, from all 5 Arabidopsis chromosomes, was plotted vs. a range of measurement bin sizes. The correlations provided here represent the 10 of the stronger relationships: (a) Retrotransposons vs. CCSs. (b) Indels vs. SNPs. (c) Retrotransposons vs. 180 bp repeats. (d) 180 bp repeats vs. CCSs. (e) Genes vs. retrotransposons (f) Genes vs. CCSs. The broken red lines represents the critical values corresponding to $P=0.05$ and obtained by simulation. (Insert) Mean correlation vs. measurement bin size for the envelope of all 15 comparisons. Here the solid red lines represent the critical values corresponding to $P=0.05$.

increased bin size indicated that in Arabidopsis a component of this correlation could be attributed to large-scale chromosomal features. At the same time, we could not exclude the possibility that the persistence of this correlation at small scales might not be attributable to localized interactions, but rather to methodological artifact since the positional cloning used to detect both SNPs and indels for the TAIR database might have allowed selection bias. 
We also observed that the densities of genes and retrotransposons exhibited statistically significant and strong negative correlation, a finding consistent with previous observations with transposable elements. This has been explained by a presumed selection against the disruption of gene expression by transposable elements[10]. As with the other associations discussed here, it was difficult to reconcile explanations based upon localized processes with the decreased strength of the correlation at small measurement scales. The hypothesis that large-scale chromosomal structure could influence the spatial distribution of a variety of chromosomal elements seemed to provide a more plausible explanation.

\section{Conclusion}

We have demonstrated that the physical distributions of genomic elements within Arabidopsis chromosomes were highly heterogeneous, yet shared a common distributional pattern. The density profiles of each element exhibited low-frequency fluctuations indicative of regional clustering, and these density profiles tended to correlate with each other at large measurement scales. This was the dominant pattern underlying the positional distributions of all 6 genomic elements examined. Localized cis-interactions between different elements had a comparatively minor, if any, influence on the intrachromosomal distribution of genomic elements. This study demonstrated an additional hierarchy of eukaryotic genomic organization that was both common to a diverse set of genomic elements, and associated with major chromosomal features.

\section{Methods \\ Data abstraction}

The Arabidopsis thaliana data used in this study was obtained from a variety of sources: The positions of genes were obtained from the National Center for Biotechnology Information (NCBI) website http:// www.ncbi.nlm.nih.gov/mapview/ and localization of indels and SNPs was provided by TAIR http://arabidop sis.org/. TIGR provided sequence information for Arabidopsis pseudochromosomes http://www.tigr.org/tdb/ $\underline{\mathrm{e} 2 \mathrm{k} 1 / \mathrm{ath} 1 /)}$ ) as well as for retrotransposons and CCSs http://www.tigr.org/tdb/e2k1/plant.repeats/. Sequences for the $180 \mathrm{bp}$ repeats were obtained from AtRepBase http://nucleus.cshl.org/protarab/AtRepBase.htm.

Localization of retrotransposons, CCSs and the $180 \mathrm{bp}$ repeats was performed by running BLASTN as provided at the TIGR website. In order to be as inclusive as possible, the stringency of these matches was kept low; as well, filters were used to exclude redundant matches. We thus accessed the positions of 29,826 genes, 1,732 indels, 20,008 SNPs, 20,564 retrotransposons, 14,064 tandem repeats, and 3,896 CCSs.
Since TAIR and TIGR act as repositories for the publicly available Arabidopsis data, a number of different methods had been used by many different investigators to identify the positions of the various genomic elements: SNPs and indels were identified by positional cloning of the available bacterial artificial chromosomes (BACs)[21], and by the large-scale analysis of expressed sequence tags from different accessions of Arabidopsis[22].

In our analysis the 5 Arabidopsis chromosomes were divided into non-overlapping, equal-sized, sequential bins and the numbers of $p$-termini for the structure of interest were enumerated for each bin. A range of bin sizes was employed, from 10 to 1,000 kb. Density profiles for the individual structures thus obtained were subjected to the additional analyses detailed below.

\section{Spectral analysis}

Density profiles for each genomic feature were parsed into $50 \mathrm{~kb}$ bins and padded with zeros to a length of 1024 data points. A $15 \%$ split-cosine-bell taper was employed on the data at the beginning and end of each sequence, the mean was subtracted, the data de-trended, and a 15-point Hamming data window was employed for data smoothing. The spectral density of each structure was calculated using a Cooley-Tukey fast Fourier transform and then normalized. The spectral densities were averaged over all 5 Arabidopsis chromosomes and then plotted $v$ s. frequency. As a control for these analyses we performed Monte Carlo simulations for a random (Poisson) distributed genomic element within five chromosomes with a density comparable to that observed with genes. These data were then processed as above to yield a spectral density. A more extensive set of simulations was done to estimate the critical values for the amplitude of individual peaks expected from a random distribution of elements, and with parameters to emulate both the chromosomal densities of elements and the lengths of the individual chromosomes.

\section{Correlation analysis}

The Pearson correlation coefficient $r$ was calculated between different density profiles over a range of bin sizes ( 10 to $1000 \mathrm{~kb}$ ), and then plotted $v$ s. bin size. We also performed scatter plots between the density profiles to exclude pronounced nonlinear relationships between variables (data not provided). Because $r^{2}$ is additive, whereas $r$ is not, the quadratic mean was used to average correlations from the 5 Arabidopsis chromosomes. Critical values for the quadratic means were estimated by Monte Carlo simulations, based upon the premise of a $t$-distribution for the quantity, $r \sqrt{(n-2) /\left(1-r^{2}\right)}$, where $n$ represents the number of data points[23]. 


\section{List of abbreviations used}

SNPs - single nucleotide polymorphisms; CCSs - conserved centromeric sequences; NCBI - National Center for Biotechnology Information; TAIR - The Arabidopsis Information Resource; TIGR - The Institute for Genomic Research; AtRepBase - Arabidopsis genomic repeat database; BACs - bacterial artificial chromosomes; $P$ value probability value; NS - not significant; vs. - versus; bp base pairs; kb - kilobases; HLA - human leukocyte associated antigens; GC - guanidine cytosine; BLASTN - basic local alignment search tool for nucleotides ; PCR polymerase chain reaction; $r$ - Pearson correlation coefficient.

\section{Authors' contributions}

WSK conceived this study, conducted the spectral and correlation analyses, and drafted the manuscript. BPS participated in the design of the study, and conducted the bioinformatic data abstraction. Both authors read and approved the final manuscript.

\section{Acknowledgements}

The authors thank Dr. Miguel Andrade for his helpful advice in the preparation of this manuscript.

\section{References}

I. Copenhaver GP, Nickel K, Kuromori T, Benito M-I, Kaul S, Lin X, Bevan M, Murphy G, Harris B, Parnell LD, et al.: Genetic definition and sequence analysis of Arabidopsis centromeres. Science 1999, 286:2468-2474.

2. Hall AE, Keith KC, Hall SE, Copenhaver GP, Preuss D: The rapidly evolving field of plant centromeres. Curr Opin Plant Biol 2004, 7(2): $108-114$.

3. Sachidanandam R, Weissman D, Schmidt SC, Kakol JM, Stein LD, Marth G, Sherry S, Mullikin JC, Mortimore BJ, Willey DL, et al.: A map of human genome sequence variation containing $\mathbf{I . 4 2}$ million single nucleotide polymorphisms. Nature 200I, 409:928-933.

4. Kendal WS: An exponential dispersion model for the distribution of human single nucleotide polymorphisms. Mol Biol Evol 2003, 20(4):579-590.

5. Kendal WS: A scale invariant clustering of genes on human chromosome 7. BMC Evol Biol 2004, 4(I):3.

6. Lercher MJ, Urrutia AO, Hurst LD: Clustering of housekeeping genes provides a unified model of gene order in the human genome. Nat Genet 2002, 3 I (2): I80- I83.

7. Lercher MJ, Urrutia AO, Pavlicek A, Hurst LD: A unification of mosaic structures in the human genome. Hum Mol Genet 2003, I 2(19):24I I-24I5.

8. Roy PJ, Stuart JM, Lund J, Kim SK: Chromosomal clustering of muscle-expressed genes in Caenorhabditis elegans. Nature 2002, 4 I 8(690I):975-979.

9. Longman-Jacobsen N, Williamson JF, Dawkins RL, Gaudieri S: In polymorphic genomic regions indels cluster with nucleotide polymorphism: Quantum Genomics. Gene 2003, 3 I 2:257-26I.

10. Wright SI, Agrawal N, Bureau TE: Effects of Recombination Rate and Gene Density on Transposable Element Distributions in Arabidopsis thaliana. Genome Res 2003, 13(8): 1897-1903.

II. van Driel R, Fransz PF, Verschure PJ: The eukaryotic genome: a system regulated at different hierarchical levels. J Cell Sci 2003, I I 6(20):4067-4075.

12. Rhee SY, Beavis W, Berardini TZ, Chen G, Dixon D, Doyle A, GarciaHernandez M, Huala E, Lander G, Montoya M, et al:: The Arabidopsis Information Resource (TAIR): a model organism database providing a centralized, curated gateway to Arabidopsis biology, research materials and community. Nucl Acids Res 2003, $31(I): 224-228$.
13. Ouyang S, Buell CR: The TIGR Plant Repeat Databases: a collective resource for the identification of repetitive sequences in plants. Nucl Acids Res 2004, 32(9000 I):D360-363.

14. The arabidopsis genome initiative: Analysis of the genome sequence of the flowering plant Arabidopsis thaliana. Nature 2000, 408(68 I4):796-8I5.

15. Theologis A, Ecker JR, Palm CJ, Federspiel NA, Kaul S, White O, Alonso J, Altafi H, Araujo R, Bowman CL, et al: Sequence and analysis of chromosome I of the plant Arabidopsis thaliana. Nature 2000, 408(68|4):8I6-820.

16. Lin X, Kaul S, Rounsley S, Shea TP, Benito MI, Town CD, Fujii CY, Mason T, Bowman CL, Barnstead M, et al:: Sequence and analysis of chromosome 2 of the plant Arabidopsis thaliana. Nature 1999, 402(6763):761-768.

17. Mayer K, Schuller C, Wambutt R, Murphy G, Volckaert G, Pohl T, Dusterhoft A, Stiekema W, Entian KD, Terryn N, et al.: Sequence and analysis of chromosome 4 of the plant Arabidopsis thaliana. Nature 1999, 402(6763):769-777.

18. Tabata S, Kaneko T, Nakamura Y, Kotani H, Kato T, Asamizu E, Miyajima N, Sasamoto S, Kimura T, Hosouchi T, et al.: Sequence and analysis of chromosome 5 of the plant Arabidopsis thaliana. Nature 2000, 408(68 I 4):823-826.

19. Salanoubat M, Lemcke K, Rieger M, Ansorge W, Unseld M, Fartmann B, Valle G, Blocker H, Perez-Alonso M, Obermaier B, et al.: Sequence and analysis of chromosome 3 of the plant Arabidopsis thaliana. Nature 2000, 408(68 I 4):820-822.

20. Longman-Jacobsen N, Williamson JF, Dawkins RL, Gaudieri S: In polymorphic genomic regions indels cluster with nucleotide polymorphism: Quantum Genomics. Gene 2003, 3 I 2:257-26I.

21. Jander G, SR N, SD R, DF B, IM L, RL L: Arabidopsis map-based cloning in the post-genome era. Plant Physiol 2002, I 29(2):440-450.

22. Schmid KJ, Sorensen TR, Stracke R, Torjek O, Altmann T, MitchellOlds T, Weisshaar B: Large-Scale Identification and Analysis of Genome-Wide Single-Nucleotide Polymorphisms for Mapping in Arabidopsis thaliana. Genome Res 2003, I 3(6a): I 250- 1257.

23. Zar JH: Biostatistical analysis. 2nd edition. Englewood Cliffs, N. J.: Prentice-Hall, Inc; 1984.
Publish with BioMed Central and every scientist can read your work free of charge

"BioMed Central will be the most significant development for disseminating the results of biomedical research in our lifetime. "

Sir Paul Nurse, Cancer Research UK

Your research papers will be:

- available free of charge to the entire biomedical community

- peer reviewed and published immediately upon acceptance

- cited in PubMed and archived on PubMed Central

- yours - you keep the copyright

Submit your manuscript here:

http://www.biomedcentral.com/info/publishing_adv.asp
BioMedcentral 